Diagnostics 2011, 1, 53-76; doi:10.3390/diagnostics1010053

Article

\title{
Bayesian Estimation of Combined Accuracy for Tests with Verification Bias
}

\section{Lyle D. Broemeling}

Broemeling \& Associates Inc., 1023 Fox Ridge Road, Medical Lake, WA 99022, USA;

E-Mail: broemeli2@aol.com

Received: 31 October 2011; in revised form: 30 November 2011 / Accepted: 5 December 2011 /

Published: 15 December 2011

\begin{abstract}
This presentation will emphasize the estimation of the combined accuracy of two or more tests when verification bias is present. Verification bias occurs when some of the subjects are not subject to the gold standard. The approach is Bayesian where the estimation of test accuracy is based on the posterior distribution of the relevant parameter. Accuracy of two combined binary tests is estimated employing either "believe the positive" or "believe the negative" rule, then the true and false positive fractions for each rule are computed for two tests. In order to perform the analysis, the missing at random assumption is imposed, and an interesting example is provided by estimating the combined accuracy of CT and MRI to diagnose lung cancer. The Bayesian approach is extended to two ordinal tests when verification bias is present, and the accuracy of the combined tests is based on the ROC area of the risk function. An example involving mammography with two readers with extreme verification bias illustrates the estimation of the combined test accuracy for ordinal tests.
\end{abstract}

Keywords: Bayesian; inverse probability weighting; verification bias; risk score; combined test accuracy

\section{Introduction}

This article introduces the reader to the methodology of measuring the accuracy of several medical tests that are administered to the patient. Our main focus is on measuring the accuracy of a combination of two or more tests. For example, to diagnose type 2 diabetes, the patient is given a 
fasting blood glucose test, which is followed by an oral glucose tolerance test. What is the accuracy (true and false positive fractions) of this combination of two tests? Or, in order to diagnose coronary artery disease, the subject's history of chest pain is followed by an exercise stress test. Still another example is for the diagnosis of prostate cancer, where a digital rectal exam is followed by measuring PSA (prostate specific antigen). The reader is referred to Johnson, Sandmire, and Klein [1] for a description of additional examples of multiple tests to diagnose a large number of diseases, including heart disease, diabetes, lung cancer, breast cancer, etc.

In many scenarios, it is common practice to administer one or more tests to diagnose a given condition and we will explore two avenues. One case where it is common to administer several tests is in standard medical practice, and the other is an experimental situation where one test is compared to a standard medical test. An example of the latter is that MRI is now being studied as an alternative to standard mammography, as a means to diagnose breast cancer.

There are many studies that assess the accuracy of the combination of two or more tests. Two tests for the diagnosis of a disease measure different aspects or characteristics of the same disease. In the case of diagnostic imaging, two modalities have different qualities (resolution, contrast, and noise), thus, although they are imaging the same scene, the information is not the same from the two sources. When this is the case, the accuracy of the combination of two modalities is of paramount importance. For example, the accuracy of the combination of mammography and scintimammography for suspected breast cancer has been reported by Buscombe, Cwikla, Holloway, and Hilson [2]. Another study for diagnosing breast cancer was performed by Berg, Gutierrez, et al. [3] who measured the accuracy of mammography, clinical examination, ultrasound, and MRI in a preoperative assessment of the disease, The accuracy of each modality and various combinations of the modalites were measured. When investigating metastasis to the lymph nodes in lung cancer, Van Iverhagen, Brakel, and Heijenbrok et al. [4] measured the accuracy of ultrasound and CT and the combination of two. Ultrasound conveys different information about metastasis compared to CT, but the combination of the two might provide a more accurate diagnosis than each separately. For an example of the diagnosis of head and neck cancer, Pauleit, Zimmerman, Stoffels et al. [5] used two nuclear medicine modalities,

${ }^{18}$ F-FET PET and ${ }^{18}$ F-FDG PET to assess the extent of the disease and estimated the accuracy of each and combined. On the other hand, Schaffler, Wolf, Schoelinast et al. [6] evaluated pleural abnormalities with CT and ${ }^{18}$ F-FDG PET and the combination of the two.

\section{Measuring the Combined Accuracy of Two Binary Tests}

What is the optimal way to measure the accuracy for combination of two binary tests? Pepe ([7], p. 207) presents two approaches: (1) believe the positive rule, or BP, where a positive test score on a subject occurs when one or the other of the two tests is scored positive; and (2) believe the negative rule, or $\mathrm{BN}$, where a subject is scores positive if both tests are scored positive. Pepe also provides some properties about these rules, namely:

a. The BP rule increases sensitivity relative to the two binary tests, but increases the FPF (false positive fraction), but by no more than the sum of the two false positive fractions FPF1 + FPF2. The false positive fraction of test 1 and test 2 are denoted by FPF1 and FPF2 respectively; 
b. The $\mathrm{BN}$ rule decreases the false positive rate relative to the false positive rates of the two tests, but at the same time, decreases the sensitivity, however, the sensitivity remains above TPF $1+$ TPF $2-1$. Note that the true positive fractions for the two tests are designated by TPF1 and TPF2.

Thus, with the BP rule the combined test is scored positive if one or the other or both of the two are scored positive, but, on the other hand, with the $\mathrm{BN}$ rule the combined test is scored positive if both tests are positive.

\section{Verification Bias}

Verification bias is present when some of the subjects are not subject to the gold standard, thus, the disease status of some of the patients is not known. Consider an example involving the exercise stress test to diagnose heart disease. Among those that test positive, some will undergo coronary angiography to confirm the diagnosis. On the other hand, among those that test negative, very few will be subject to the gold standard. Using only those cases that are verified will lead to biased estimates of test accuracy both of the true and false positive rates, thus, alternative methods based on the missing at random assumptions will be derived from a Bayesian viewpoint.

When assessing the accuracy of two tests, the design in many cases is paired. When two tests are used to assess the patient's condition, true and false positive fractions will be estimated assuming the $\mathrm{BP}$ (believe the positive) and BN (believe the negative) rules. For example, two modalities (e.g., CT and MRI) are imaging the same patients and the two images would be expected to be quite similar. Another case of a paired design is for two readers who are imaging the same set of patients with the same imaging device. One expects the information gained from the two paired sources to be highly correlated, and in the case of two paired readers, agreement between the two is also of interest. The experimental layout for a paired study when verification bias is present, namely:

A Good introduction to statistical methods for estimating test accuracy with verification bias is Zhou et al. ([8], p. 307), who use maximum likelihood estimation for the study below.

Table 1. Two binary scores with verification bias.

$\mathrm{Y}_{1}=1,0$
\begin{tabular}{|c|c|c|c|c|}
\hline $\mathrm{V}=1$ & $\mathrm{Y}_{2}=1$ & $\mathrm{Y}_{2}=0$ & $\mathrm{Y}_{2}=1$ & $\mathrm{Y}_{2}=0$ \\
\hline $\mathrm{D}=1$ & $s_{11}$ & $s_{10}$ & $s_{01}$ & $s_{00}$ \\
\hline $\mathrm{D}=0$ & $r_{11}$ & $r_{10}$ & $r_{01}$ & $r_{00}$ \\
\hline $\mathrm{V}=0$ & $u_{11}$ & $u_{10}$ & $u_{01}$ & $u_{00}$ \\
\hline Total & $m_{11}$ & $m_{10}$ & $m_{01}$ & $m_{00}$ \\
\hline
\end{tabular}

The two binary tests are $Y_{1}$ and $Y_{2}$, where 1 and 0 designate positive and negative tests respectively. For those subjects who are verified for disease $\mathrm{V}=1$, while those who are not verified are denoted with $\mathrm{V}=0$.

Note that the number of subjects verified under the gold standard, when both tests are positive, is $s_{11}+r_{11}$, among which $s_{11}$ had the disease and $r_{11}$ did not have the disease, and also the number that 
were not verified under the gold standard when both tests are positive is $u_{11}$ etc., also note that the total number of subjects is $\sum_{i, j=0}^{i, j=1} m_{i j}=m_{. .}$.

\section{Posterior Distribution of Combined Test Accuracy}

The following derivation is based on the MAR assumption, namely

$$
P\left[V=1 \mid Y_{1}, Y_{2}, D\right]=P\left[V=1 \mid Y_{1}, Y_{2}\right]
$$

Thus, the probability a subject's disease status is verified depends only on the outcomes of the two tests, and not on other considerations. Derivations below follow to some extent Chapter 10 of Zhou, Obuchowski, and McClish ${ }^{8}$.

Suppose the unknown parameters are defined as follows:

$$
\varphi_{i j}=P\left[D=1 \mid Y_{1}=i, Y_{2}=j\right]
$$

and

$$
\theta_{i j}=P\left[Y_{1}=i, Y_{2}=j\right]
$$

for $\mathrm{i}, \mathrm{j}=0,1$.

Also let

$$
\varphi_{i .}=P\left[D=1 \mid Y_{1}=i\right]
$$

and

$$
\varphi_{. j}=P\left[D=1 \mid Y_{2}=j\right]
$$

where $\mathrm{i}, \mathrm{j}=0,1$.

The likelihood for the parameters is

$$
L(\theta, \varphi) \propto \prod_{i=0}^{i=1} \prod_{j=0}^{j=1} \varphi_{i j}^{s_{i j}}\left(1-\varphi_{i j}\right)^{r_{i j}} \prod_{i=0}^{i=1} \prod_{j=0}^{j=1} \theta_{i j}^{m_{i j}}
$$

Assuming an improper prior distribution for the parameters, the posterior distributions are

$$
\varphi_{i j} \sim \operatorname{beta}\left(s_{i j}, r_{i j}\right)
$$

for $\mathrm{i}, \mathrm{j}=0,1$, and the $\theta_{i j}$ are distributed Dirichlet with parameter vector $\left(m_{00}, m_{01}, m_{10}, m_{11}\right)$.

The improper prior imposed on the parameters is given by the density

$$
\xi(\theta, \varphi) \propto \prod_{i=0}^{i=1} \prod_{j=0}^{j=1} \theta_{i j}^{-1} \varphi_{i j}^{-1}
$$

Note that

$$
\varphi_{1 .} \sim \operatorname{beta}\left(s_{1 .}, r_{1}\right)
$$

and

$$
\varphi .1 \sim \operatorname{beta}\left(s_{.1}, r_{.1}\right)
$$


where

$$
S_{1 .}=S_{11}+S_{10}
$$

and

$$
r_{1 .}=r_{11}+r_{10}
$$

Note that if a uniform prior is assumed, one should adjust the posterior distributions for the phis and thetas by adding a one to the beta and Dirichlet hyper parameters given in formulas (6) and (7).

The main parameters of interest are the true positive fraction and the false positive fraction for the two tests, thus for the first test

$$
\operatorname{tpf} f_{1}=P\left[Y_{1}=1 \mid D=1\right]
$$

and is given by Bayes theorem as

$$
t p f_{1}=\varphi_{1 .} \theta_{1 .} /\left(\varphi_{1 .} \theta_{1 .}+\varphi_{0 .} \theta_{0 .}\right)
$$

where the $\phi_{i}$. are given by (8.19) and

$$
\theta_{1 .}=\theta_{11}+\theta_{10}
$$

As for test 1, the false positive fraction is given by

$$
f p f_{1}=\left(1-\varphi_{1 .}\right) \theta_{1 .} /\left(1-\varphi_{1} \theta_{1 .}-\varphi_{0} \theta_{0}\right)
$$

With regard to test 2 , the true positive fraction is

$$
\operatorname{tpf} 2=\varphi_{.1} \theta_{.1} /\left(\varphi_{.1} \theta_{.1}+\varphi_{.0} \theta_{.0}\right)
$$

and the false positive fraction is

$$
\operatorname{fpf} 2=\left(1-\varphi_{.1}\right) \theta_{.1} /\left(1-\varphi_{.1} \theta_{.1}+\varphi_{.0} \theta_{.0}\right)
$$

The main focus of this section is on measuring the accuracy of the combined test in the presence of verification bias of both tests using the BN (believe the negative) and BP (believe the positive) principles.

Assume the BP principle is in effect, then the true positive fraction for the combined test is

$$
\text { tpfbp }=P\left[Y_{1}=1 \text { or } Y_{2}=1 \mid \mathrm{D}=1\right]
$$

while

$$
f p f b p=P\left[Y_{1}=1 \text { or } Y_{2}=1 \mid \mathrm{D}=0\right]
$$

Now assume the $\mathrm{BN}$ assumption is in effect, then the true positive fraction is

$$
\text { tpfbn }=P\left[Y_{1}=1, Y_{2}=1 \mid \mathrm{D}=1\right]
$$

while

$$
f p f b n=P\left[Y_{1}=1, Y_{2}=1 \mid \mathrm{D}=0\right]
$$

The above four accuracy measures can be expressed as follows: for the BP assumption,

$$
t p f b p=\left(\varphi_{11} \theta_{11}+\varphi_{01} \theta_{01}+\varphi_{10} \theta_{10}\right) / P[D=1]
$$

and

$$
f p f b p=\left(\left(1-\varphi_{11}\right) \theta_{11}+\left(1-\varphi_{01}\right) \theta_{01}+\left(1-\varphi_{10}\right) \theta_{10}\right) / P[D=0]
$$


where

$$
\left.P[D=1]=\varphi_{11} \theta_{11}+\varphi_{01} \theta_{01}+\varphi_{10} \theta_{10}+\varphi_{00} \theta_{00}\right)
$$

For the $\mathrm{BN}$ assumption

$$
\text { tpfbn }=\varphi_{11} \theta_{11} / P[D=1]
$$

and

$$
f p f b n=\left(1-\varphi_{11}\right) \theta_{11} / P[D=0]
$$

Formulas (16)-(19) measure the combined accuracy of two binary tests assuming MAR and assuming an improper prior. If a uniform prior distribution is assumed for the $\varphi_{i j}$ and $\theta_{i j}$, adjust the hyper parameters in formulas (6) and (7) accordingly. For additional information about estimating accuracy with verification bias see Zhou [9,10] and Zhou and Castelluccio [11].

\section{Example of MRI and CT to Assess Risk of Lung Cancer}

Consider the hypothetical results of two correlated binary tests when verification bias is present as given below. The first test $\mathrm{Y}_{1}$ give the results for a CT determination of lung cancer risk, where a 0 indicates a small risk and a 1 a high risk of lung cancer, while the second test $\mathrm{Y}_{2}$ is a determination of lung cancer risk using MRI. The patients where $\mathrm{D}=0$ do not have lung cancer.

Table 2. CT and lung cancer risk.

$\mathrm{Y}_{1}=1,0$
\begin{tabular}{|c|c|c|c|c|}
\hline $\mathrm{V}=1$ & $\mathrm{Y}_{2}=1$ & $\mathrm{Y}_{2}=0$ & $\mathrm{Y}_{2}=1$ & $\mathrm{Y}_{2}=0$ \\
\hline $\mathrm{D}=1$ & $s_{11}=14$ & $s_{10}=12$ & $s_{01}=9$ & $s_{00}=3$ \\
\hline $\mathrm{D}=0$ & $r_{11}=4$ & $r_{10}=9$ & $r_{01}=13$ & $r_{00}=18$ \\
\hline $\mathrm{V}=0$ & $u_{11}=7$ & $u_{10}=8$ & $u_{01}=9$ & $u_{00}=10$ \\
\hline Total & $m_{11}=25$ & $m_{10}=29$ & $m_{01}=31$ & $m_{00}=31$ \\
\hline
\end{tabular}

Using BUGS CODE 1, an analysis that determines the accuracy of the CT and MRI separately and for the combined accuracy is executed with 45,000 observations, with a burn in of 5,000 and a refresh of 100. The list statement of the code gives the data for this example, assuming an improper prior distribution:

\section{BUGS CODE 1}

model; \{

\# two binary tests verification bias

\# accuracy of combined tests

g00 dgamma(m00,2)

g01 dgamma $(\mathrm{m} 01,2)$

g10 dgamma $(\mathrm{m} 10,2)$

g11 dgamma $(\mathrm{m} 11,2)$

$\mathrm{h}<-\mathrm{g} 00+\mathrm{g} 01+\mathrm{g} 10+\mathrm{g} 11$

th $00<-$ g00/h

th $01<-$ g0 $01 / \mathrm{h}$

th $10<-$ g $10 / h$ 
th $11<-$ g $11 / \mathrm{h}$

ph00 dbeta(s00,r00)

ph01 dbeta $(\mathrm{s} 01, \mathrm{r} 01)$

ph10 dbeta(s10,r10)

ph11 dbeta(s11,r11)

s1.<-s11+s10

r1.<-r11+r10

s. $1<-\mathrm{s} 01+\mathrm{s} 11$

r. $1<-\mathrm{r} 01+\mathrm{r} 11$

$\mathrm{r} 0 .<-\mathrm{r} 00+\mathrm{r} 01$

$\mathrm{s} 0 .<-\mathrm{s} 00+\mathrm{s} 01$

s. $0<-\mathrm{s} 00+\mathrm{s} 10$

r. $0<-\mathrm{r} 00+\mathrm{r} 10$

ph1. dbeta(s1.,r1.)

ph.1 dbeta(s.1,r.1)

ph0. dbeta(s0.,r0.)

ph.0 dbeta(s.0,r.0)

th $1 .<-$ th $11+$ th 10

th. $1<-$ th $01+$ th 11

th0. $<$-th $01+$ th 00

th. $0<-$ th $00+$ th 10

\# accuracy for test 1

tpf1<-ph1.*th1./pd1

fpf1<-(1-ph1.)*th1./(1-pd1)

\# $\mathrm{p}$ [D-1]

pd1<-ph1.*th1.+ph0.*th0.

\# accuracy for test 2

tpf $2<$-ph. $1 *$ th. $1 /$ pd 2

fpf $2<-(1-$ ph. 1$) *$ th. $1 /(1-$ pd2 $)$

pd $2<-$ ph. $1 *$ th. $1+$ ph. $0 *$ th. 0

\# accuracy combined tests

\# believe the positive, BP

tpfbp $<-($ ph1 $1 *$ th $11+$ ph01*th01+ph10*th10)/pd

$\# \mathrm{P}[\mathrm{d}=1]$

pd<- ph $11 *$ th $11+$ ph $10 *$ th $10+$ ph $01 *$ th $01+$ ph00*th00

fpfbp $<-((1-$ ph11)*th11+(1-ph10)*th10+(1-ph01)*th01)/(1-pd)

\# believe the negative, $\mathrm{BN}$

tpfbn<-ph11*th $11 /$ pd

fpfbn<- $(1-$ ph11)*th11/(1-pd)\}

\# CT and MRI for lung cancer risk with improper prior

\# for a uniform prior, add a one to the values in the list statement

$\operatorname{list}(\mathrm{s} 00=3, \mathrm{r} 00=18, \mathrm{~s} 01=9, \mathrm{r} 01=13, \mathrm{~s} 10=12, \mathrm{r} 10=9, \mathrm{~s} 11=14, \mathrm{r} 11=4$, 
$\mathrm{m} 00=31, \mathrm{~m} 01=31, \mathrm{~m} 10=29, \mathrm{~m} 11=25)$

\# activate initial values from the specification tool with the gen inits button

Note the above code closely follows the derivation given in formulas (1)-(19). This analysis can be executed by downloading the code from http://medtestacc.blogspot.com.

Table 3. Posterior analysis for the CT and MRI determination of lung cancer risk with verification bias.

\begin{tabular}{|c|c|c|c|c|c|c|}
\hline Parameter & Mean & SD & Error & $\mathbf{2 ~ 1 / 2}$ & Median & $\mathbf{9 7} \mathbf{1 / 2}$ \\
\hline fpf1 & 0.2864 & 0.06201 & $<0.0001$ & 0.1725 & 0.284 & 0.4141 \\
\hline fpf2 & 0.3809 & 0.0668 & $<0.0001$ & 0.253 & 0.3799 & 0.5144 \\
\hline fpfbn & 0.0880 & 0.0697 & $<0.0001$ & 0.0264 & 0.0829 & 0.1795 \\
\hline fpfbp & 0.5766 & 0.0653 & $<0.0001$ & 0.4559 & 0.5777 & 0.7021 \\
\hline tpf1 & 0.6755 & 0.0712 & $<0.0001$ & 0.5305 & 0.6779 & 0.8074 \\
\hline tpf2 & 0.6007 & 0.0737 & $<0.0001$ & 0.4538 & 0.6018 & 0.7414 \\
\hline tpfbn & 0.3663 & 0.0694 & $<0.0001$ & 0.2367 & 0.3645 & 0.5081 \\
\hline tpfbp & 0.9165 & 0.0439 & $<0.0001$ & 0.8131 & 0.9233 & 0.9812 \\
\hline
\end{tabular}

The results of the analysis show that the false positive fractions for the two modalities are fairly high being 0.286 and 0.38 for CT and MRI respectively, but on the other hand, the true positive fractions for the two modalities are somewhat low at 0.6755 and 0.60 respectively for CT and MRI. It is also observed that the MCMC errors for all parameters are less than 0.0001 and the posterior distributions of all parameters appear to be symmetric about the posterior mean. Assuming the BN rule, the true and false positive fractions are estimated as 0.3663 and 0.0880 , but as for the BP rule, the corresponding estimates (based on the posterior mean) are 0.9165 and 0.5766 ! The fact that both modalities are not very accurate is reflected in the estimated combined accuracies for the BN and BP rules. At first glance, the BP rule is encouraging in that the true positive fraction is 0.9165 , but the false positive fraction is also large as 0.5766 . The $\mathrm{BN}$ rule gives a low estimate of 0.0880 for the false positive rate, but also a low estimate of 0.3663 for the true positive fraction. The posterior density of the true positive fraction for the BP rule is depicted below. Note what rule the user adopts depends on their personal preference.

Figure 1. Posterior density of true positive fraction BP rule.

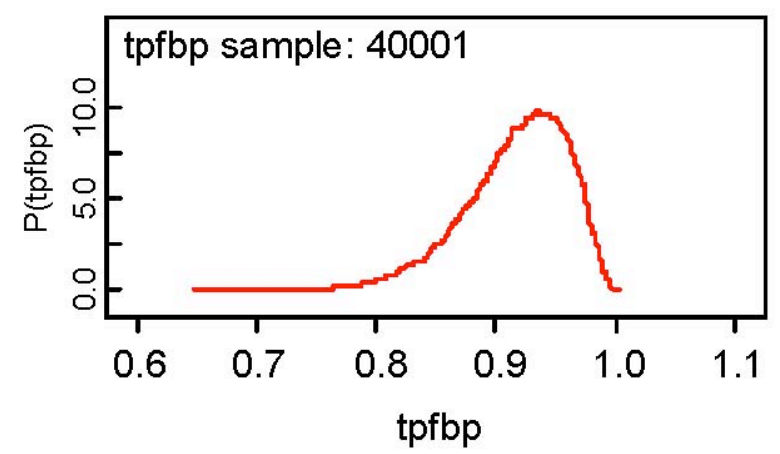




\section{Extreme Verification Bias}

The reader is referred to Pepe ([7], p. 180) and Broemeling ([12], p. 166) for a description of many studies for those cases where when the test is positive, the subject is referred to the gold standard (verification of disease status), but if the test is negative the subject is not referred to the gold standard. In such situations, the true and false positive rates are not estimable. Consider the following hypothetical example given by above in Table 2, where MRI and CT are jointly used to detect lung cancer, where the results of CT are given by the first test and those of MRI given by $Y_{2}$. Note when the subject is verified with $\mathrm{D}=0$, the patient does not have lung cancer.

Table 4. CT and MRI for lung cancer risk with extreme verification bias.

$\mathrm{Y}_{1}=1,0$
\begin{tabular}{|c|c|c|c|c|}
\hline $\mathrm{V}=1$ & $\mathrm{Y}_{2}=1$ & $\mathrm{Y}_{2}=0$ & $\mathrm{Y}_{2}=1$ & $\mathrm{Y}_{2}=0$ \\
\hline $\mathrm{D}=1$ & $s_{11}=14$ & $s_{10}=12$ & $s_{01}=9$ & $s_{00}=0$ \\
\hline $\mathrm{D}=0$ & $r_{11}=4$ & $r_{10}=9$ & $r_{01}=13$ & $r_{00}=0$ \\
\hline $\mathrm{V}=0$ & $u_{11}=0$ & $u_{10}=0$ & $u_{01}=0$ & $u_{00}=21$ \\
\hline Total & $m_{11}=18$ & $m_{10}=21$ & $m_{01}=22$ & $m_{00}=21$ \\
\hline
\end{tabular}

The results of Table 2 have been modified so that all patients are referred to the gold standard (biopsy) except those where both tests are negative. There are 82 patients, of which 61 are verified for disease, namely the 18 who test positive when both CT and MRI are positive, 21 patients who test positive with CT but negative with MRI, and lastly 22 patients who test positive with MRI and negative with $\mathrm{CT}$.

When both tests are negative, 21 patients are not subject to the gold standard. Since they were not verified, one does not know their disease status $(D=0$ or $D=1)$, thus one does not know the fraction of patients with disease, and the true and false positive fractions cannot be estimated. This is a case where it is not possible to estimate the true and false positive fractions for either test. However, not all is lost because other measures of test accuracies for both tests can be estimated.

Consider the detection probability for $\mathrm{CT}$ namely $\mathrm{P}\left[Y_{1}=1, \mathrm{D}=1\right]$, then the estimated detection probability is $26 / 82=0.317$, while the detection probability of MRI is estimated as $23 / 82=0.280$. In lieu of the true and false positive fractions, the detection probability and the false referral probability for both tests aid in the estimation of the test accuracy of the combined tests. For example, the false referral probability for $\mathrm{CT}$ is $\mathrm{P}\left[Y_{1}=1, \mathrm{D}=0\right]$ which is estimated as $13 / 82=0.158$, and for MRI the false referral probability is estimated as $17 / 82=0.2073$.

It is interesting to note that the detection probability of a test is expressed as

$$
\mathrm{DP}=\rho \mathrm{TPF}
$$

and the false referral probability as

$$
\mathrm{FRP}=(1-\rho) \mathrm{FPF}
$$

where $\rho=\mathrm{P}[\mathrm{D}=1]$, is the probability of disease, and TPF and FPF are the true and false positive fractions respectively. From a given study the probability of disease cannot be estimated, however the true positive fractions and be compared with the ratio: 


$$
\operatorname{rtpf}(\mathrm{ct} / \mathrm{mri})=D P_{1} / D P_{2}
$$

where $D P_{1}$ is the detection probability of the first test and $D P_{2}$ the detection probability for the second test. In a similar way, the false positive fractions can be compared by the ratio:

$$
\operatorname{rfpf}(\mathrm{ct} / \mathrm{mri})=F R P_{1} / F R P_{2}
$$

where $F R P_{1}$ is the false referral probability for the first test and $F R P_{2}$ the false referral probability of the second. Of course, the Bayesian approach will determine the posterior distribution of these quantities.

It is interesting to observe that the individual true and false positive fractions cannot be estimated, but that the true and false positive fractions of two tests can be compared for studies with extreme verification bias.

How is the accuracy of the combined tests estimated? Can one employ the BP (believe the positive) and $\mathrm{BN}$ rules to estimate the accuracy of the combined tests? The answer is no!

Consider the BP rule and refer to Table 4, where the true positive fraction of the combined tests is measured by $\mathrm{P}\left[Y_{1}=1\right.$ or $\left.Y_{2}=1 \mid \mathrm{D}=1\right]$, however, the disease frequency cannot be estimated. On the other hand, the ratio of the true positive fraction for the BP rule relative to the true positive fraction of the $\mathrm{BN}$ rule can be measured by

$$
\begin{gathered}
\text { tpfbp/tpfbn }=\mathrm{P}\left[Y_{1}=1 \text { or } Y_{2}=1 \mid \mathrm{D}=1\right] / \mathrm{P}\left[Y_{1}=1 \text { and } Y_{2}=1 \mid \mathrm{D}=1\right] \\
=P\left[Y_{1}=1 \text { or } Y_{2}=1 \text { and } D=1\right] / P\left[Y_{1}=1 \text { and } Y_{2}=1 \text { and } D=1\right]
\end{gathered}
$$

From Table 4 for the CT-MRI study of lung cancer risk, a naïve estimate of this quantity is $35 / 14=2.5$, which implies the true positive fraction for the BP rule is approximately 2.5 times larger than the true positive fraction for the $\mathrm{BN}$ rule.

In a similar way the false positive fraction for the BP rule relative to the false positive fraction of the $\mathrm{BN}$ rule can be measured as

$$
\begin{gathered}
\mathrm{fpfbp} / / \mathrm{fpfbn}=\mathrm{P}\left[Y_{1}=1 \text { or } Y_{2}=1 \mid \mathrm{D}=0\right] / \mathrm{P}\left[Y_{1}=1 \text { and } Y_{2}=1 \mid \mathrm{D}=0\right] \\
=P\left[Y_{1}=1 \text { or } Y_{2}=1 \text { and } D=0\right] / P\left[Y_{1}=1 \text { and } Y_{2}=1 \text { and } D=0\right]
\end{gathered}
$$

Referring to Table 4 provides an estimate of $26 / 4=6.5$ for comparing the false positive fractions for the two rules, implying the false positive rate for the BP rule is six and half times larger that of the BN rule.

Extreme verification bias does not provide sufficient information to estimate the usual measures of test accuracy, however, in lieu of those measures, it is possible to assess the accuracy of two binary tests (with extreme verification bias) by: (1) the detection probabilities; (2) the false referral probabilities and (3) the ratio of the true positive fraction for the BP rule relative to that of the BN rule, and (4) the ratio of the false positive fraction of the BP rule relative to that of the BN rule.

\section{Bayesian Analysis for Extreme Verification Bias}

The foundation of the Bayesian analysis given by formulas (1)-(19) which are now expanded to determine the posterior distribution of the detection probabilities and false referral probabilities of both tests.

With regard to estimating the combined accuracy of the two tests in the presence of extreme verification bias, the posterior distributions of the ratio of the true positive fraction of the BP relative to 
that of the $\mathrm{BN}$ rule is determined as is the ratio of the false positive fraction of the $\mathrm{BP}$ rule relative to that of the $\mathrm{BN}$ rule.

For the detection probability of the first test

$$
\mathrm{DP} 1=\mathrm{P}\left[Y_{1}=1, D=1\right]=\theta_{1} \varphi_{1} .
$$

where the summation is taken over the missing subscript denoted by a period. Note the $\theta_{i j}$ and $\varphi_{i j}$ are defined by formulas (1) and (2), thus in a similar way it can be shown that

$$
\mathrm{DP} 2=\mathrm{P}\left[Y_{2}=1, D=1\right]=\theta_{.1} \varphi_{.1}
$$

As for the false referral probabilities, the one for the first test is

$$
\mathrm{FRP} 1=P\left[Y_{1}=1, D=0\right]=\left(1-\varphi_{1 .}\right) \theta_{1}
$$

It can also be shown that for the second test,

$$
\mathrm{FRP} 2=P\left[Y_{2}=1, D=0\right]=\left(1-\varphi_{.1}\right) \theta_{.1}
$$

Referring to formulas (1), (2), (24), and (25), the ratio of the true positive fraction of the BP rule relative to that of the $\mathrm{BN}$ rule is

$$
\mathrm{rtpfbp} / \mathrm{tpfbn}=\left[\theta_{11} \varphi_{11}+\theta_{10} \varphi_{10}+\theta_{01} \varphi_{01}\right] /\left[\theta_{11} \varphi_{11}\right]
$$

As for the ratio of the false positive fraction of the BP rule relative to that of the $\mathrm{BN}$ rule, it can be shown

$$
\mathrm{rfpfbp} / \mathrm{fpfbn}=\left[\theta_{11}\left(1-\varphi_{11}\right)+\theta_{10}\left(1-\varphi_{10}\right)+\theta_{01}\left(1-\varphi_{01}\right)\right] /\left[\theta_{11}\left(1-\varphi_{11}\right)\right]
$$

Formulas (26)-(31) measure the accuracy of two combined binary tests when extreme verification bias is present.

\section{Bayesian Analysis for Risk of Lung Cancer}

BUGS CODE 1 is amended with the following WinBUGS $®$ code.

For the detection probabilities, the statements are:

$$
\text { DP1<-th1.*ph1. }
$$

and

$$
\text { DP2<-th. } 1 * \text { ph. } 1
$$

For the false referral probabilities of the two tests:

$$
\text { FRP1<-(1-ph1.)*th1. }
$$

and

$$
\text { FRP2<-(1-ph.1)*th.1. }
$$

The above code corresponds to formulas (26)-(20).

Refer to formula (30) for the ratio of the true positive fraction of the $\mathrm{BP}$ rule relative to that of the $\mathrm{BN}$ rule, thus the following:

$$
\text { Rtpfbptpfbn<-(th11*ph11+th10*ph10+th01*ph01)/(th11*ph11) }
$$


Refer to formula (31) for the ratio of the false positive fraction of the BP rule to that of the BN rule, thus the code is

$$
\text { Rfpfbpfpfbn<-(th11*(1-ph11)+th10*(1-ph10)+th01*(1-ph01))/(th11*(1-ph11)) }
$$

The list statement for the data is given by:

$$
\begin{aligned}
\operatorname{list}(\mathrm{s} 00=1, \mathrm{r} 00=1, \mathrm{~s} 01=9, \mathrm{r} 01=13, \mathrm{~s} 10 & =12, \mathrm{r} 10=9, \mathrm{~s} 11=14, \mathrm{r} 11=4, \mathrm{~m} 00=21, \mathrm{~m} 01=22, \\
\mathrm{~m} 10 & =21, \mathrm{~m} 11=18)
\end{aligned}
$$

which assumes an improper prior density for all parameters. Although $\mathrm{s} 00=1$ and $\mathrm{r} 00=1$ are zero (when the two tests are negative all patients are referred to the gold standard), I put a one, which does not affect the analysis.

The analysis for the CT-MRI determination of lung cancer risk with extreme verification bias is based on the amended BUGS CODE 1 by generating 55,000 samples from the posterior distributions of the detection and false referral probabilities of both CT and MRI. Also computed are the posterior distributions of the ratio of the true and false positive fractions of the BP rule relative to that of the $\mathrm{BN}$ rule. I used a burn in of 5,000 observations with a refresh of 100 to give.

Table 5. Bayesian analysis for extreme verification bias (callout).

\begin{tabular}{|c|c|c|c|c|c|c|}
\hline Parameter & Mean & SD & Error & $\mathbf{2 ~ 1 / 2}$ & Median & $\mathbf{9 7 ~ 1 / 2}$ \\
\hline DP1 & 0.3169 & 0.0508 & $<0.0001$ & 0.22 & 0.3155 & 0.4206 \\
\hline DP2 & 0.2805 & 0.0493 & $<0.0001$ & 0.1891 & 0.2787 & 0.3823 \\
\hline FRP1 & 0.1586 & 0.0400 & $<0.0001$ & 0.0882 & 0.1559 & 0.2411 \\
\hline FRP2 & 0.2074 & 0.0446 & $<0.0001$ & 0.127 & 0.2051 & 0.3016 \\
\hline Ratio TPF & 2.618 & 0.5922 & 0.0019 & 1.774 & 2.515 & 4.503 \\
\hline Ratio FPF & 8.346 & 5.629 & 0.0168 & 3.187 & 6.902 & 22.01 \\
\hline
\end{tabular}

One notices the skewness of the posterior distribution of the ratio of the false positive fraction of the $\mathrm{BP}$ rule relative to the $\mathrm{BN}$ rule and the skewness is evident from Figure 2 below. I would use 6.902 the posterior median as a point estimate of the ratio.

Figure 2. Posterior density of the ratio of the false positive fraction. BP relative to BN.

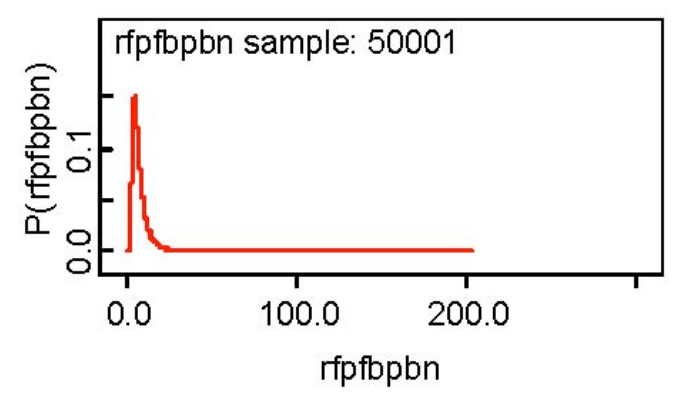

The other posterior distributions appear to be symmetric about the posterior mean. The detection probability of the two tests are similar, but the false referral probability of CT is somewhat less than that of MRI and the TPF of the BP rule appears to be 2.61 times that of the BN rules. On the other hand, the false positive fraction of the $\mathrm{BP}$ rule is 6.9 times that of the $\mathrm{BN}$ rule. 
Recall that the true and false positive fractions of the two rules are not known, thus, it is difficult to interpret these ratios. The detection probability of a test is somewhat related to the true positive fraction in that one would favor a test with a higher detection probability. Also, of course, one would favor a test with lower false referral probability, thus, the overall conclusion about test comparison is to favor the CT determination of lung cancer risk. As for the accuracy of the combined test, the two ratios one for the true positive fraction and one for the false positive fraction provide the relevant information. Should the accuracy be based on the BP or the BN rule? The BP rule gives a larger true positive fraction, but unfortunately a much larger false positive fraction.

\section{Verification Bias for Two Ordinal Tests}

When analyzing the accuracy of combined tests when the tests are ordinal, a somewhat different approach is taken, where the overall accuracy is measured by the area of the ROC curve of the risk score. The risk scores are the probability of disease of each patient, usually determined. An informative presentation of the risk score is given by Pepe ([7], p. 271).

Our general methodology is based on inverse probability weighting which transforms the original table of observations with verification (See Table), to an imputed table. Such ideas will be explained in a later section, but the reader is referred to Pepe ([7], p.172) and Broemeling ([13], p. 279) for additional details of the inverse probability weighting approach for estimating the accuracy of tests with verification bias.

The analysis of assessing the accuracy of two tests is now expanded to include two ordinal tests $T_{1}$ and $T_{2}$, where the general layout is given by Table 5 . As before the $s_{i}$ denote the number of patients for the 9 events when $\mathrm{D}=1$, while the $r_{i}$ represent the number of cases for the nine events when $\mathrm{D}=0$. The total number of observations is $\sum_{i=1}^{i=9} m_{i}$. Each test has three values, but of course the general situation has a similar scenario.

Table 5. Two ordinal tests with verification bias.

$T_{1}=1,2,3$
\begin{tabular}{|c|c|c|c|c|c|c|c|c|c|}
\hline$T_{2}$ & 1 & 2 & 3 & 1 & 2 & 3 & 1 & 2 & 3 \\
\hline $\mathrm{D}=1$ & $s_{1}$ & $s_{2}$ & $s_{3}$ & $s_{4}$ & $s_{5}$ & $s_{6}$ & $s_{7}$ & $s_{8}$ & $s_{9}$ \\
\hline $\mathrm{D}=0$ & $r_{1}$ & $r_{2}$ & $r_{3}$ & $r_{4}$ & $r_{5}$ & $r_{6}$ & $r_{7}$ & $r_{8}$ & $r_{9}$ \\
\hline $\mathrm{V}=0$ & $u_{1}$ & $u_{2}$ & $u_{3}$ & $u_{4}$ & $u_{5}$ & $u_{6}$ & $u_{7}$ & $u_{8}$ & $u_{9}$ \\
\hline Total & $m_{1}$ & $m_{2}$ & $m_{3}$ & $m_{4}$ & $m_{5}$ & $M_{6}$ & $m_{7}$ & $m_{8}$ & $M_{9}$ \\
\hline
\end{tabular}

In what is to follow, the posterior distribution of the ROC area of the two ordinal tests is developed, which is to be followed by an explanation of inverse probability weighting, and lastly the use of the risk score to asses the accuracy of the combined tests is explained.

\section{Posterior Distribution of the ROC Areas for Two Ordinal Tests with Verification Bias}

Recall from formulas (1) and (2) that

$$
\varphi_{i j}=P\left[D=1 \mid Y_{1}=i, Y_{2}=j\right] \text { and } \theta_{i j}=P\left[Y_{1}=i, Y_{2}=j\right]
$$


thus

$$
\varphi_{i} \cdot=P\left[D=1 \mid Y_{1}=i\right]
$$

and

$$
\varphi_{. i}=P\left[D=1 \mid Y_{2}=i\right]
$$

for $i=1,2,3$, then assuming an improper prior density,

$$
\varphi_{i .} \sim \operatorname{beta}\left(s_{i .}, r_{i .}\right)
$$

and

$$
\varphi_{. i} \sim \operatorname{beta}\left(s_{. i}, r_{. i}\right)
$$

The improper prior density used here is the reciprocal of the parameters over the relevant region of the parameter space.

If uniform prior is appropriate, add a 1 to the hyper parameters of the $\varphi_{i}$ and $\theta_{i}$ for $\mathrm{i}=1,2,3$.

Note that

$$
\begin{gathered}
s_{1 .}=s_{1}+s_{2}+s_{3} \\
s_{.1}=s_{1}+s_{4}+s_{7} \\
r_{1 .}=r_{1}+r_{2}+r_{3} \text { etc. }
\end{gathered}
$$

Also, let

$$
\theta_{1}=P\left[Y_{1}=1, Y_{2}=1\right]
$$

and

$$
\theta_{9}=P\left[Y_{1}=3, Y_{2}=3\right]
$$

In addition, let

$$
\theta_{1 .}=\theta_{1}+\theta_{2}+\theta_{3}
$$

thus

$$
\theta_{1 .}=P\left[Y_{1}=1\right]
$$

Also let

$$
\theta_{3}=\theta_{3}+\theta_{6}+\theta_{9}
$$

then

$$
\theta_{3}=P\left[Y_{2}=3\right] \text { etc. }
$$

In order to compute the area under the ROC curve, Bayes theorem is used to compute

$$
\alpha 1_{i}=\varphi_{i .} \theta_{i .} / t \alpha 1
$$

where $\mathrm{i}=1,2,3$, and

$$
t \alpha 1=\sum_{i=1}^{i=3} \varphi_{i} \theta_{i}
$$

Note that

$$
\alpha 1_{i}=P\left[Y_{1}=i \mid D=1\right]
$$


And in a similar manner

$$
\beta 1_{i}=\left(1-\varphi_{i .}\right) \theta_{i .} / t \beta 1
$$

where

$$
t \beta 1=\sum_{i=1}^{i=3}\left(1-\varphi_{i .}\right) \theta_{i}
$$

thus

$$
\begin{gathered}
\beta 1_{i}=P\left[Y_{1}=i \mid \mathrm{D}=0\right] \\
\alpha 2_{i}=P\left[Y_{2}=i \mid \mathrm{D}=1\right]
\end{gathered}
$$

and

$$
\beta 2_{i}=P\left[Y_{2}=i \mid \mathrm{D}=0\right]
$$

can be defined. The ROC area for the first test is

$$
A_{1}=A_{11}+A_{12}
$$

where

$$
A_{11}=\alpha 1_{2} \beta 1_{1}+\alpha 1_{3}\left(\beta 1_{1}+\beta 1_{2}\right)
$$

and

$$
2 A_{12}=\sum_{i=1}^{i=3} \alpha 1_{i} \beta 1_{i}
$$

Of course, a similar expression holds for the ROC area of test 2. After determining the posterior distribution of the ROC areas of the two tests, the accuracy of the combined tests will be measured by the ROC area of the risk score. In order to illustrate Bayesian inference for estimating the accuracy of two ordinal tests, consider the following example, two readers are diagnosing breast cancer. They are both employed by a university cancer center and they both use a three-point scale to diagnose the disease, where a 1 indicates definitely no lesion is seen in the mammogram, a 2 denotes there is a possibility that a lesion is present, and a 3 signifies that a lesion is definitely present The diseased patients in fact have breast cancer and the non-diseased definitely do not have breast cancer.

Table 6. Diagnosing breast cancer with two readers.

$T_{1}=1,2,3$
\begin{tabular}{|c|c|c|c|c|c|c|c|c|c|}
\hline$T_{2}$ & 1 & 2 & 3 & 1 & 2 & 3 & 1 & 2 & 3 \\
\hline $\mathrm{D}=1$ & $s_{1}$ & $s_{2}$ & $s_{3}$ & $s_{4}$ & $s_{5}$ & $s_{6}$ & $s_{7}$ & $s_{8}$ & $s_{9}$ \\
& 8 & 26 & 51 & 43 & 81 & 94 & 117 & 140 & 208 \\
\hline $\mathrm{D}=0$ & $r_{1}$ & $r_{2}$ & $r_{3}$ & $r_{4}$ & $r_{5}$ & $r_{6}$ & $r_{7}$ & $r_{8}$ & $r_{9}$ \\
& 101 & 105 & 83 & 67 & 72 & 40 & 41 & 30 & 4 \\
\hline $\mathrm{V}=0$ & $u_{1}$ & $u_{2}$ & $u_{3}$ & $u_{4}$ & $u_{5}$ & $u_{6}$ & $u_{7}$ & $u_{8}$ & $u_{9}$ \\
& 2 & 18 & 62 & 14 & 83 & 67 & 63 & 40 & 108 \\
\hline Total & $m_{1}$ & $m_{2}$ & $m_{3}$ & $m_{4}$ & $m_{5}$ & $m_{6}$ & $m_{7}$ & $m_{8}$ & $m_{9}$ \\
& 111 & 149 & 196 & 124 & 236 & 201 & 221 & 210 & 320 \\
\hline
\end{tabular}


The total number of patients is $\sum_{i=1}^{i=9} m_{i}=1,768$, and for each of the nine categories patients are not referred to the gold standard, for example. For example, when $T_{1}=T_{2}=1,109$ are referred to the gold standard and 2 are not, while if $T_{1}=T_{2}=2,83$ are not referred, while 153 are subject the gold standard. What are the ROC areas of the two tests? The following code is based on the previous development of the posterior distributions of the relevant parameters (given by formulas (36)-(50)) and appears below.

\section{BUGS CODE 2}

model;

\# hypothetical data set

\# two tests for staging melanoma

\# one rater is a surgeon the other a dermatologist

\# ratings are: stage 1 , stage 2 , stage 3

\# similar to Zhou([8],p347) on CT and MRI

\{

for (i in 1:9) $\{\mathrm{ph}[\mathrm{i}] \sim \operatorname{dbeta}(\mathrm{s}[\mathrm{i}], \mathrm{r}[\mathrm{i}])\}$

for (i in 1:9) $\{\mathrm{g}[\mathrm{i}] \sim \operatorname{dgamma}(\mathrm{m}[\mathrm{i}], 2)\}$

$\mathrm{ms}<-\operatorname{sum}(\mathrm{g}[])$

for (i in $1: 9)\{$ theta $[\mathrm{i}]<-\mathrm{g}[\mathrm{i}] / \mathrm{ms}\}$

theta1.<- theta[1]+theta[2]+theta[3]

theta2.<- theta[4]+theta[5]+theta[6]

theta3.<- theta[7]+theta[8]+theta[9]

theta. $1<-$ theta[1]+theta[4]+theta[7]

theta. $2<-$ theta[2]+theta[5]+theta[8]

theta.3<- theta[3]+theta[6]+theta[9]

$\mathrm{s} 1 .<-\mathrm{s}[1]+\mathrm{s}[2]+\mathrm{s}[3]$

$\mathrm{s} 2 .<-\mathrm{s}[4]+\mathrm{s}[5]+\mathrm{s}[6]$

$\mathrm{s} 3 .<-\mathrm{s}[7]+\mathrm{s}[8]+\mathrm{s}[9]$

s. $1<-\mathrm{s}[1]+\mathrm{s}[4]+\mathrm{s}[7]$

s. $2<-\mathrm{s}[2]+\mathrm{s}[5]+\mathrm{s}[8]$

s. $3<-\mathrm{s}[3]+\mathrm{s}[6]+\mathrm{s}[9]$

r1. $<-r[1]+r[2]+r[3]$

r2. $<-r[4]+r[5]+r[6]$

r3. $<-r[7]+r[8]+r[9]$

r. $1<-\mathrm{r}[1]+\mathrm{r}[4]+\mathrm{r}[7]$

r. $2<-r[2]+r[5]+r[8]$

r.3<-r[3]+r[6]+r[9]

\# the prob $\mathrm{D}=1$ given $\mathrm{Y} 1=1$

ph1. dbeta(s1.,r1.)

ph2. d dbeta(s2.,r2.)

ph3. dbeta(s3.,r3.)

ph.1 dbeta(s.1,r.1) 


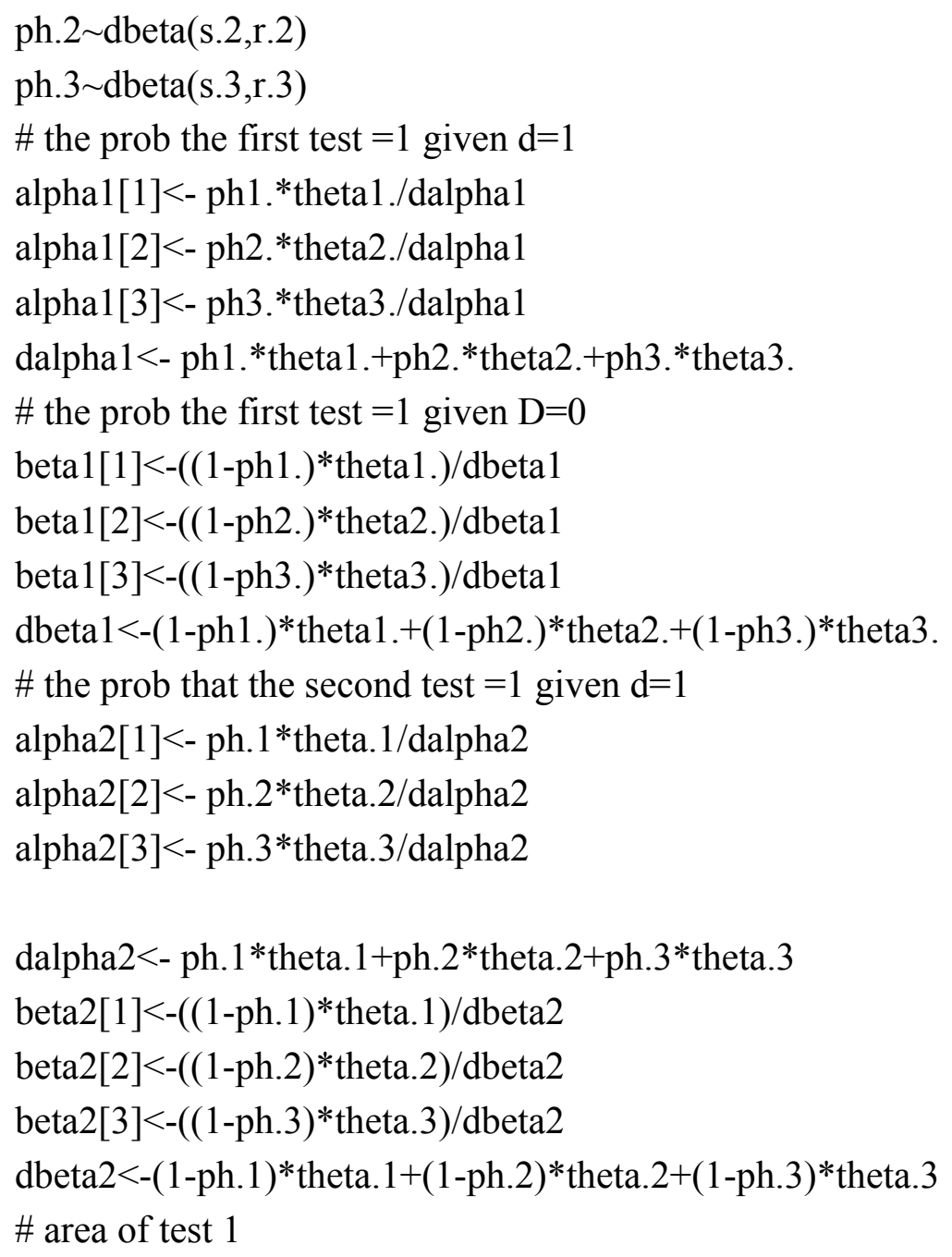

A $1<-\mathrm{A} 11+\mathrm{A} 12$

A11<- alpha1[2]*beta1[1]+alpha1[3]*( beta1[1]+beta1[2])

A12<- (alpha1[1]*beta1[1]+alpha1[2]*beta1[2]+alpha1[3]*beta1[3])/2

\# area of test 2

A2 <- A21+A22

A21<- alpha2[2]*beta2[1]+alpha2[3]*( beta2[1]+beta2[2])

A22<- (alpha2[1]*beta2[1]+alpha2[2]*beta2[2]+alpha2[3]*beta2[3])/2

$\mathrm{d}<-\mathrm{A} 1-\mathrm{A} 2$

\}

\# two readers

\# assume an improper prior

\# see Table 6

$\operatorname{list}(\mathrm{r}=\mathrm{c}(101,105,83,67,72,40,41,30,4), \mathrm{s}=\mathrm{c}(8,26,51,43,81,94,117,140,208)$,

$\mathrm{m}=\mathrm{c}(111,149,196,124,236,201,221,210,320))$

\# the initial values are activated from the specification tool by clicking on the gen inits button.

The code closely follows formulas (36)-(50), and the analysis is executed with 55,000 samples from the posterior distribution of the parameters, with a burn in of 5,000 and a refresh of 100 and the results are reported in Table 7. 
Table 7. Bayesian analysis for ROC areas of two readers.

\begin{tabular}{|c|c|c|c|c|c|c|}
\hline Parameter & Mean & SD & Error & $21 / 2$ & Median & $971 / 2$ \\
\hline A1(reader 1) & 0.7867 & 0.0119 & $<0.00001$ & 0.763 & 0.787 & 0.8097 \\
\hline A2(reader 2) & 0.6351 & 0.0145 & $<0.00001$ & 0.6062 & 0.6352 & 0.6633 \\
\hline
\end{tabular}

Reader 1 appears more accurate that reader 2. It should be noted that the gold standard is biopsy of the tissue from the suspected lesion. The MCMC errors are quite "small" and give one confidence that the simulation is providing accurate estimates of the accuracy.

The main goal of the analysis is to estimate the combined accuracy of the two readers nd compare it the individual estimated ROC areas given by Table 7 .

\section{Inverse Probability Weighting}

Pepe ([7], p. 171) describes an interesting variation on estimating test accuracy with verification bias by the inverse probability weighting technique, and for further information, see Begg and Greenes [14]. Briefly this method involves constructing an imputed data table from the observed data table with verification bias. Consider the observed data table (Table 6) with verification bias. For each pair of values $\left(T_{1}, T_{2}\right)$, the cell entries for $s_{i}$ and $r_{i}$ are multiplied by the inverse of the verification rate. For example, when $\left(T_{1}, T_{2}\right)=(1,1)$ the verification rate is $109 / 111$, thus for $s_{1}, 8$ is replaced $8(111 / 109)=8.146$ and for $r_{1}$, the number 101 is replaced by $101(111 / 109)=102.85$. If this is done for the remaining 8 events, the observed Table 6 is replaced by the selected table below:

Table 8. Diagnosing breast cancer with two readers imputed table via inverse probability weighting.

$T_{1}=1,2,3$
\begin{tabular}{|c|c|c|c|c|c|c|c|c|}
\hline 1 & 2 & 3 & 1 & 2 & 3 & 1 & 2 & 3 \\
\hline$s_{1}$ & $s_{2}$ & $s_{3}$ & $s_{4}$ & $s_{5}$ & $s_{6}$ & $s_{7}$ & $s_{8}$ & $s_{9}$ \\
8 & 30 & 75 & 48 & 125 & 141 & 164 & 164 & 314 \\
\hline$r_{1}$ & $r_{2}$ & $r_{3}$ & $r_{4}$ & $r_{5}$ & $r_{6}$ & $r_{7}$ & $r_{8}$ & $r_{9}$ \\
103 & 119 & 121 & 76 & 111 & 60 & 57 & 37 & 6 \\
\hline$u_{1}$ & $u_{2}$ & $u_{3}$ & $u_{4}$ & $u_{5}$ & $u_{6}$ & $u_{7}$ & $u_{8}$ & $u_{9}$ \\
0 & 0 & 0 & 0 & 0 & 0 & 0 & 0 & 0 \\
\hline$m_{1}$ & $m_{2}$ & $m_{3}$ & $m_{4}$ & $m_{5}$ & $m_{6}$ & $m_{7}$ & $m_{8}$ & $m_{9}$ \\
111 & 149 & 196 & 124 & 236 & 201 & 221 & 210 & 320 \\
\hline
\end{tabular}

The imputed table has no verification bias and the ROC areas of the two tests based on this table are the same as those based on Table 6 , the table with verification bias. In general it can be shown for binary tests that the true and false positive fractions are the same, for those based on the observed table and those based on the selected table without verification bias. See Pepe ([7], p. 172), thus, inverse probability weighting is appropriate for two ordinal tests with verification bias. It is seen the effect of inverse probability weighting is to convert a table with verification bias into a table without. The presentation is continued by developing the risk score to assess the accuracy of two ordinal tests with verification bias, 
where the basic idea is to compute the ROC of the risk score. For additional information about estimating the test accuracy of ordinal tests with verification bias using the ROC area, see Gray et al. [15].

\section{The Risk Score}

The risk score is the probability that $\mathrm{D}=1$, which is the value assigned to all patients in the study. For example, for each of the 1,768 patients in the melanoma staging study portrayed in Table 8 , the probability of disease is assigned and the ROC area computed. The ROC area of the risk score is the combined accuracy of the two ordinal tests with verification bias.

The risk score is defined as

$$
\mathrm{RS}(\mathrm{Y})=\mathrm{P}[\mathrm{D}=1 \mid \mathrm{Y}]
$$

and has the property that it is a monotone function of the likelihood ratio. Simply stated, the risk score assigns a probability of disease to each study subject.

The risk score (51) has the same ROC curve as the likelihood ratio and the same optimal properties. Observe that

$$
\begin{aligned}
& \mathrm{RS}(\mathrm{Y})=\mathrm{P}[\mathrm{D}=1 \mid \mathrm{Y}] \\
& =\mathrm{P}[\mathrm{Y} \mid \mathrm{D}=1] \mathrm{P}[\mathrm{D}=1] / \mathrm{P}[\mathrm{Y}] \\
& =\mathrm{P}[\mathrm{Y} \mid \mathrm{D}=1] \mathrm{P}[\mathrm{D}=1] /\{\mathrm{P}[\mathrm{Y} \mid \mathrm{D}=1] \mathrm{P}[\mathrm{D}=1]+\mathrm{P}[\mathrm{Y} \mid \mathrm{D}=0] \mathrm{P}[\mathrm{D}=0]\} \\
& =\mathrm{LR}(\mathrm{Y}) \mathrm{P}[\mathrm{D}=1] /\{\mathrm{LR}(\mathrm{Y}) \mathrm{P}[\mathrm{D}=1]+\mathrm{P}[\mathrm{D}=1]\}
\end{aligned}
$$

which shows that the risk score is a monotone increasing function of the likelihood ratio, which implies that the ROC curve of risk score is the same as that of the likelihood ratio. For our purposes the risk score will be used to measure the accuracy of combined tests, namely, using the area of the ROC curve of the risk score. Pepe ([7], p. 274) shows the utility of logistic regression for finding the ROC curve of the risk score. Note that the following statements show why.

Suppose the risk score is expressed as

$$
\operatorname{logitP}[\mathrm{D}=1 \mid \mathrm{Y}]=\gamma+g(\lambda, Y)
$$

where $\mathrm{g}$ is a known function, then:

(a) the parameter $\lambda$ can be estimated, even for retrospective designs in which the sampling depends on $\mathrm{D}$, and

(b) the function $\mathrm{g}$ is optimal for determining the ROC curve of the risk function.

From a practical point of view, logistic regression can be used to determine the ROC curve of the risk function, but it should be noted that finding a suitable function $\mathrm{g}$ can be a challenge. After all, $g$ can be a complicated non-linear function of $\lambda$ and/ or $Y$, but it would be convenient if $g$ is linear in the test scores $\mathrm{Y}$. In order to estimate the logistic regression function or risk score, Broemeling ([13], p. 474), takes a Bayesian approach.

For the example of Table 8 , where two readers are diagnosing breast cancer, the risk score is computed by logistic regression using the following code. 


\section{BUGS CODE 3}

model;

\# logistic regression

\{

for( $\mathrm{i}$ in $1: \mathrm{N})\{\mathrm{d}[\mathrm{i}] \sim \operatorname{dbern}(\operatorname{theta}[\mathrm{i}])\}$

for $(\mathrm{i}$ in $1: \mathrm{N})\{\operatorname{logit}($ theta[i] $)<-\mathrm{b}[1]+\mathrm{b}[2] * \mathrm{~T} 1[\mathrm{i}]+\mathrm{b}[3] * \mathrm{~T} 2[\mathrm{i}]\}$

\# prior distributions

for $(i$ in $1: 3)\{b[i] \sim \operatorname{dnorm}(0.000, .0001)\}$

\}

In order to accommodate the data, a list statement must be added, which consists of three vectors, each of dimension 1,768, corresponding to the number of patients in the study: The vectors $T_{1}$ and $T_{2}$ will consist of the values 1,2 , or 3 corresponding to Table 8 , while a $\mathrm{d}$ vector will consists of zeros and ones, where a 1 indicates the patient has melanoma and a 0 indicates no melanoma, thus, the first 1,078 components of $d$ consist of a one, while the remaining 690 consist of zeros. After execution of the BUGS CODE 3, with 45,000 samples from the posterior distribution, with a burn in of 5,000, and a refresh of 100, the posterior distribution of coefficients of the logistic regression is given in Table 9.

Table 9. Posterior distribution of the logistic regression the risk score.

\begin{tabular}{|c|c|c|c|c|c|c|}
\hline Parameter & Mean & SD & Error & $\mathbf{2 ~ 1 / 2}$ & Median & $\mathbf{9 7 ~ 1 / 2}$ \\
\hline $\mathrm{b}[1]$ & -4.947 & 0.2859 & 0.00173 & $-5,515$ & -4.943 & -4.395 \\
\hline $\mathrm{b}[2]$ & 1.688 & 0.0851 & $<0.0001$ & 1.524 & 1.687 & 1.857 \\
\hline $\mathrm{b}[3]$ & 0.8946 & 0.0804 & $<0.0001$ & 0.7373 & 0.894 & 1.053 \\
\hline
\end{tabular}

The posterior distributions indicate the coefficients are not zero and are important in determining the risk score of each patient, but the main interest is in the vector theta (of dimension 1,768) where the median of each component is used as the risk score. There were 9 distinct risk scores: $0.086,0.187$, $0.36,0.336,0.5542,0.733,0.7542,0.8704$, and 0.9426. Therefore, I computed the ROC using the basic formula from Broemeling ([12], p. 58) for the ROC area of a test with ordinal responses.

Using BUGS CODE 4, I computed the ROC area of the risk score via BUGS CODE 4 with posterior mean 0.842 with a $95 \%$ credible interval of $(0.8238,0.8608)$. (Also, the ROC area was computed with a non-parametric technique with SPSS with the same result as the Bayesian). Thus, using the risk score, the estimated ROC area is 0.842 compared to a ROC area of 0.78 for the surgeon and 0.63 for the dermatologist, thus, the accuracy of the combining results of two readers is more than the individual accuracies. Consider the posterior analysis:

Table 10. Posterior analysis of combined accuracy breast cancer example with two readers.

\begin{tabular}{|c|c|c|c|c|c|c|}
\hline Parameter & Mean & SD & Error & $\mathbf{2 ~ 1 / 2}$ & Median & $\mathbf{9 7 ~ 1 / 2}$ \\
\hline A1 & 0.8099 & 0.0106 & $<0.00001$ & 0.7885 & 0.8101 & 0.8308 \\
\hline A2 & 0.0657 & 0.0030 & $<0.00001$ & 0.0599 & 0.0656 & 0.0716 \\
\hline auc & 0.8428 & 0.0094 & $<0.00001$ & 0.8238 & 0.843 & 0.8608 \\
\hline
\end{tabular}


Based on BUGS CODE 4, the analysis is executed with 55,000 observations with a burn in of 5,000 and a refresh of 100 . A2 is the probability of a tie, and auc is the ROC area. Small MCMC errors for the parameters are evident, and the posterior distribution of all parameters appear to be symmetric. The code below is explained with explanatory remarks indicated by \#.

\section{BUGS CODE 4}

\# Area under the curve

\# Ordinal values

\# Nine values

model;

\{

\# generate Dirichlet distribution

g11 dgamma(a11,2)

g12 dgamma(a12,2)

g13 dgamma(a13,2)

g14 dgamma(a14,2)

g15 dgamma(a15,2)

g16 dgamma(a16,2)

g17 dgamma(a17,2)

g18 dgamma(a18,2)

g19 dgamma(a19,2)

g01 dgamma(a01,2)

g02 dgamma(a02,2)

g03 dgamma(a03,2)

g04 dgamma(a04,2)

g05 dgamma $(\mathrm{a} 05,2)$

g06 dgamma(a06,2)

g07 dgamma $(\mathrm{a} 07,2)$

g08 dgamma(a08,2)

g09 dgamma $(a 09,2)$

$\mathrm{g} 1<-\mathrm{g} 11+\mathrm{g} 12+\mathrm{g} 13+\mathrm{g} 14+\mathrm{g} 15+\mathrm{g} 16+\mathrm{g} 17+\mathrm{g} 18+\mathrm{g} 19$

$\mathrm{g} 0<-\mathrm{g} 01+\mathrm{g} 02+\mathrm{g} 03+\mathrm{g} 04+\mathrm{g} 05+\mathrm{g} 06+\mathrm{g} 07+\mathrm{g} 08+\mathrm{g} 09$

\# posterior distribution of probabilities for response of diseased patients

theta $1<-$ g $11 / \mathrm{g} 1$

theta $2<-$ g $12 / g 1$

theta $3<-$ g $13 / g 1$

theta $4<-$ g $14 / g 1$

theta $5<-$ g $15 / g 1$

theta $6<-$ g $16 / g 1$

theta $7<$-g17/g1

theta $8<-$ g18/g1

theta $9<-$ g19/g1 
\# posterior distribution for probabilities of response of non-diseased patients

$\mathrm{ph} 1<-\mathrm{g} 01 / \mathrm{g} 0$

ph $2<$-g02/g0

$\mathrm{ph} 3<$-g03/g0

$\mathrm{ph} 4<-\mathrm{g} 04 / \mathrm{g} 0$

ph5<-g05/g0

ph6<-g06/g0

$\mathrm{ph} 7<-\mathrm{g} 07 / \mathrm{g} 0$

ph8<-g08/g0

ph9<-g09/g0

\# auc is area under ROC curve

$\# \mathrm{~A} 1$ is the $\mathrm{P}[\mathrm{Y}>\mathrm{X}]$

$\# \mathrm{~A} 2$ is the $\mathrm{P}[\mathrm{Y}=\mathrm{X}]$

auc $<-\mathrm{A} 1+\mathrm{A} 2 / 2$

A $1<-$ theta $2 *$ ph $1+$ theta $3 *($ ph $1+$ ph 2$)+$ theta $4 *($ ph $1+$ ph $2+$ ph 3$)+$

theta $5 *(\mathrm{ph} 1+\mathrm{ph} 2+\mathrm{ph} 3+\mathrm{ph} 4)+$ theta $6 *(\mathrm{ph} 1+\mathrm{ph} 2+\mathrm{ph} 3+\mathrm{ph} 4+\mathrm{ph} 5)+$

theta $7 *(\mathrm{ph} 1+\mathrm{ph} 2+\mathrm{ph} 3+\mathrm{ph} 4+\mathrm{ph} 5+\mathrm{ph} 6)+$

theta $8 *(\mathrm{ph} 1+\mathrm{ph} 2+\mathrm{ph} 3+\mathrm{ph} 4+\mathrm{ph} 5+\mathrm{ph} 6+\mathrm{ph} 7)+$

theta $9 *(\mathrm{ph} 1+\mathrm{ph} 2+\mathrm{ph} 3+\mathrm{ph} 4+\mathrm{ph} 5+\mathrm{ph} 6+\mathrm{ph} 7+\mathrm{ph} 8)$

A2<- theta $1 *$ ph $1+$ theta $2 *$ ph $2+$ theta $3 *$ ph $3+$ theta $4 *$ ph 4

+ theta $5 *$ ph $5+$ theta $6 *$ ph $6+$ theta $7 *$ ph $7+$ theta $8 *$ ph $8+$ theta $9 *$ ph 9

\}

\#see Table 8

\# diagnosing breast cancer with two readers

\# verification bias

\# improper prior

$\operatorname{list}(\mathrm{a} 11=8, \mathrm{a} 12=30, \mathrm{a} 13=75, \mathrm{a} 14=48, \mathrm{a} 15=.125, \mathrm{a} 16=164, \mathrm{a} 17=141, \mathrm{a} 18=173, \mathrm{a} 19=314, \mathrm{a} 01=103, \mathrm{a} 02=119, \mathrm{a}$ $03=121, \mathrm{a} 04=76, \mathrm{a} 05=111, \mathrm{a} 06=57, \mathrm{a} 07=60, \mathrm{a} 08=37, \mathrm{a} 09=6$ )

\# initial values

$\operatorname{list}(\mathrm{g} 11=1, \mathrm{~g} 12=1, \mathrm{~g} 13=1, \mathrm{~g} 14=1, \mathrm{~g} 15=1, \mathrm{~g} 16=1, \mathrm{~g} 17=1, \mathrm{~g} 18=1, \mathrm{~g} 19=1, \mathrm{~g} 01=1, \mathrm{~g} 02=1, \mathrm{~g} 03=1$, $\mathrm{g} 04=1, \mathrm{~g} 05=1, \mathrm{~g} 06=1, \mathrm{~g} 07=1, \mathrm{~g} 08=1, \mathrm{~g} 09=1$ )

\section{Comments and Conclusions}

This presentation has reviewed the Bayesian methodology available for estimating the test accuracy when two or more tests are used to diagnose disease. The main focus is on tests that are subject to verification bias, that is, when some of the patients are not subject to the gold standard (are not verified for disease status). When verification bias occurs certain techniques are employed to correct for bias. If the usual estimators are calculated only for those patients that are verified for disease, the estimators are biased, thus, this article develops Bayesian procedures that "correct" for bias. By imposing the missing at random assumption, Bayesian estimators of the usual measures of test accuracy are developed. For two binary tests, 
the true and false positive fractions estimate the test accuracy, while for two ordinal tests the ROC area of the score function estimates the test accuracy of the combined tests. An interesting variation of verification bias is extreme verification bias, which is present when all of the patients that test negative with both tests are not verified for disease. In such a scenario, the true and false positive fractions cannot be estimated. However, other measures of the combined accuracy are available and easily estimated with Bayesian inference. Bayesian inference is illustrated with examples involving the diagnosis of breast and lung cancer.

There is a large literature on the subject of verification bias, and for additional recent information about the subject see [16-19]. Very little has appeared from a Bayesian viewpoint, however, Buzoianu and Kadane [20] present results on adjustment for verification bias. Broemeling [12,13] is the only book that focuses on the Bayesian approach to verification bias.

\section{References}

1. Johnson, D.; Sandmire, D.; Klein, D. Medical Tests that Can Save Your Life, Tests Your Doctor Won't Order Unless You Know To Ask; Rodale Inc.: Emmaus, PA, USA, 2004.

2. Buscombe, J.R.; Cwikla, J.B.; Holloway, B.; Hilson, A.J.W. Prediction and usefulness of combined mammography and scintimmaography in suspected primary breast cancer using ROC curves. J. Nuclear Medicine 2001, 42, 3.

3. Berg, W.A.; Gutierrez, L.; NessAlver, M.S.; Carter, W.B.; Bhargavan, M.; Lewis, R.S.; Loffe, O.B. Diagnostic accuracy of mammography, clinical examination, US and MR, imaging in preoperative assessment of breast cancer. Radiology 2004, 233, 830-849.

4. Van Overhagen, H.; Brakel, K.; Heijenbrok, M.W.; van Kasteren, J.H.L.M.; van de Moosdijk, C.N.F.; Roldann, A.C.; van Gils, A.P.; Hansen, B.E. Metastasis in supraclavicular lymph nodes in lung cancer: Assessment in palpation, US, and CT. Radiology 2004, 232, 75-80.

5. Pauleit, D.; Zimmerman, A.; Stoffels, G.; Bauer, D.; Risse, J.; Fluss, M.O.; Hamacher, K.; Coenene, H.H.; Langen, K.J. ${ }^{18}$ F-FET PET compared with ${ }^{18}$ F-FEG PET ad CT in patients with head and neck cancer. J. Nuclear Med. 2006, 47, 256-261.

6. Schaffler, G.J.; Wolf, G.W.; Schoellnast, H.; Groell, R.; Maier, A.; Smolle-Juttner, F.M.; Woltsche, M.; Fasching, G.; Nicolletti, R.; Aigner, R.M. Non-small cell lung cancer: Evaluation of pleural abnormalities on CT scans with ${ }^{18}$ F-FET PET. Radiology 2004, 231, 858-865.

7. Pepe, M.S. The Statistical Evaluation of Medical Tests for Classification and Prediction; Oxford University Press: Oxford, UK, 2004.

8. Zhou, X.H.; Obuchowski, N.A.; McClish, D.K. Statistical Methods in DiagnosticMedicine; John Wiley \& Sons Inc.: New York, NY, USA, 2002.

9. Zhou, X.H. Maximum likelihood estimators of sensitivity and specificity corrected for verification bias. Commun. Stat. Theory Methods 1993, 22, 3177-3198.

10. Zhou, X.H.; Nonparametric ML estimate of the ROC area corrected for verification bias. Biometrics 1996, 52, 310-316.

11. Zhou, X.H.; Castelluccio, P. Nonparametric analysis for the ROC curves of two diagnostic tests in the presence of non ignorable verification bias. J. Stat. Plan. Infer. 2002, 115, 193-213. 
12. Broemeling, L.D. Bayesian Biostatistics and Diagnostic Medicine; Chapman \& Hall/CRC, Taylor \& Francis: Boca Raton, FL, USA, 2007.

13. Broemeling, L.D. Advanced Bayesian Methods for Medical Test Accuracy; Chapman \& Hall/CRC, Taylor \& Francis: Boca Raton, FL, USA, 2011.

14. Begg, C.B.; Greenes, R.A. Assessment of diagnostic tests when disease verification is subject to selection bias. Biometrics 1983, 39, 207-215.

15. Gray, R.; Begg, C.; Greenes, R. Construction of receiver operating characteristic curve when disease verification is subject to selection bias, Med. Decis. Making 1984, 4, 151-164.

16. Liu, D.; Zhou, X.H. A model for adjusting for nonignorable verification bias in estimation of the ROC curve and its area with likelihood-based approach. Biometrics 2010, 66, 1119-1128.

17. Zhang, Z. Likelihood-based confidence sets for partially identified parameters. J. Stat. Plan. Infer. 2009, 139, 696-710.

18. Alonzo, T.A. Comparing accuracy in an unpaired post-market device study with incomplete disease assessment. Biom. J. 2009, 51, 491-503.

19. He, H.; Lyness, J.M.; McDermott, M.P. Direct estimation of the area under the receiver operating characteristic curve in the presence of verification bias. Stat. Med. 2009, 28, 361-376.

20. Buzoianu, M.; Kadane, J.B. Adjusting for verification bias in diagnostic test evaluation: A Bayesian approach. Stat. Med. 2008, 27, 2453-2473.

(C) 2011 by the authors; licensee MDPI, Basel, Switzerland. This article is an open access article distributed under the terms and conditions of the Creative Commons Attribution license (http://creativecommons.org/licenses/by/3.0/). 